\title{
NONVANISHING LOCAL COHOMOLOGY CLASSES ${ }^{1}$ \\ BY \\ IRA MOSKOWITZ
}

\begin{abstract}
We discuss the nonvanishing of a top-dimensional canonical cohomology class of the space $\bar{B} \mathscr{D i f f}_{\omega} M$. We treat parallelizable and odd-dimensional stably parallelizable manifolds.
\end{abstract}

Conventions. The following hold unless stated otherwise:

(1) $M$ is a closed, oriented, $n$-dimensional smooth manifold with volume form $\omega$.

(2) All $H^{*}$ and $H_{*}$ are with R-coefficients.

(3) $\operatorname{deg} f$ is the degree of $f$.

(4) $[\eta]$ is the cohomology class of the closed form $\eta$.

Introduction. Let $\mathscr{D i f f}_{\omega} M$ denote the group of volume preserving (with respect to $\omega)$ diffeomorphisms of $M$ with the $C^{\infty}$ topology. We will investigate certain local cohomology classes of $\mathscr{D i f f}_{\omega} M$, originally defined by $\mathrm{McDuff}[\mathbf{M}-1]$. These classes are related to the Gelfand-Fuks cohomology of divergence free (with respect to $\omega$ ) vector fields on $\boldsymbol{M}$. These classes also, in a certain sense, measure how $\mathscr{D i f f}_{\omega} \boldsymbol{M}$ twists $M$. In this paper we will show that the top-dimensional class in question is nonzero if $M$ is parallelizable or if $M$ is odd-dimensional and stably parallelizable.

Background. Let $\operatorname{Diff}_{\omega}^{\delta} M$ denote $\mathscr{D}_{i f} f_{\omega} M$ with the discrete topology. If $\mathscr{G}$ is a topological group, then $B \mathscr{G}$ denotes its classifying space. The inclusion $\tilde{i}$ : Diff ${ }_{\omega}^{\delta} M$ $\rightarrow$ Diff $_{\omega} M$ passes to a continuous map

$$
i: B \operatorname{Diff}_{\omega}^{\delta} M \rightarrow B \operatorname{Diff}_{\omega} M .
$$

Let $\bar{B} \mathscr{D i f f}_{\omega} M$ be the homotopy theoretic fibre of (1). This space is well-defined up to homotopy type. By the local cohomology of $\mathscr{D i f f}_{\omega} M$ we mean the (real) singular cohomology of $\bar{B} \mathscr{D}_{i f} M$.

The space $\bar{B} \mathscr{D}_{i f f_{\omega}} M$ is in its own right a classifying space. It classifies globally trivialized $M$-bundles with a flat structure. Sitting over $\bar{B} \mathscr{D}_{\text {iff }} M$ we have the universal bundle $\bar{B} \mathscr{D}_{\text {iff }} M \times M$. Let us now choose a specific model for $\bar{B} \mathscr{D i f f}_{\omega} M$ [Ma].

Let Sing $\mathscr{D}_{\text {iff }} M$ be the smooth singular complex of $\mathscr{D i f f}_{\omega} M$. Note that $\mathscr{D i f f}_{\omega} M$ acts freely on Sing $\mathscr{D}_{i f} f_{\omega} M$ by multiplication on the right, so it also acts freely on the

Received by the editors December 23, 1983. Presented at the 90th annual meeting of the AMS in Louisville, January 27, 1984.

1980 Mathematics Subject Classification. Primary 57T99, 57R32.

${ }^{1}$ This paper is part of the author's doctoral thesis. The author wishes to extend his thanks to Professor Dusa McDuff. 
geometric realization $\mid$ Sing $\mathscr{D i f f}_{\omega} M \mid$. We can now form the quotient space $\mid$ Sing $\mathscr{D i f f}_{\omega} M \mid / \operatorname{Diff}_{\omega}^{\delta} \boldsymbol{M}$. This will be our particular model for $\bar{B}$ Diff $_{\omega} M[\mathbf{M}-1]$.

Thus $\bar{B}$ Diff $_{\omega} M$ has a PL-structure in which a $k$-simplex is a smooth map $\Delta^{k} \rightarrow \mathscr{D i f f}_{\omega} M$, which is well defined up to composition on the right by an element of Diff ${ }_{\omega}^{\delta} M$. To get rid of this ambiguity we ask for the 0 -vertex to go to the identity diffeomorphism;

$$
\left(\Delta^{k}, 0\right) \rightarrow\left(\mathscr{D i f f}_{\omega} M, 1\right), \quad t \rightarrow h_{t} .
$$

We are now in a position to define a foliation $\mathscr{F}$ on $\bar{B} \mathscr{D}_{\text {iff }} \boldsymbol{M} \times M$.

To each $k$-simplex in $\bar{B} \mathscr{D}_{\text {iff }} \omega$ we can associate a foliation on $\Delta^{k} \times M$ with leaves

$$
\mathscr{L}_{m}=\left\{\left(t, h_{t}(m)\right): t \in \Delta^{k}\right\} .
$$

This foliation is the pull-back of the point foliation on $M$ by the map $f:(t, m) \rightarrow$ $h_{t}^{-1}(m)$. The foliation is volume preserving [L] and has $f^{*} \omega$ for its transverse volume form. The foliations and transverse volume forms on each $\{k$-simplex $\} \times M$ fit together to give a codimension- $n$ volume preserving (generalized) foliation $\mathscr{F}$ on $\bar{B}$ Diff $_{\omega} M \times M$ with transverse volume form $\Omega$.

For more background information see [Mo].

McDuff's classes. The cohomology class $[\Omega]$ may be decomposed via the Künneth formula. So

$$
[\Omega]=\bigoplus_{i=0}^{n}[\Omega]_{i}, \quad \text { where }[\Omega]_{i} \in H^{n-i}\left(\bar{B} \mathscr{D}_{i f f} M\right) \otimes H^{i}(M) .
$$

The class $[\Omega]_{i}$ may be written as $\sum_{j} \alpha_{n-i}^{j} \otimes \beta_{i}^{j}$, where $j$ is indexed over the rank of $H^{i}(M), \alpha_{n-i}^{j} \in H^{n-i}\left(\bar{B} \mathscr{D i f f}_{\omega} M\right)$, and $\beta_{i}^{j} \in H^{i}(M)$. Of course this representation is not unique.

We may now define the McDuff classes $c_{k}(M) \in H^{k}\left(\bar{B} \mathscr{D}_{i f f} M ; H^{n-k}(M)\right)$ by the formula

$$
c_{k}(M) \kappa=\sum_{j}\left\langle\alpha_{k}^{j}, \kappa\right\rangle \cdot \beta_{n-k}^{j},
$$

where $\kappa \in H_{k}\left(\bar{B} \mathscr{D}_{i f f} M\right)$. Note that the class $c_{k}(M)$ is independent of the choices made. In fact $c_{k}(M) \kappa$ is just, neglecting sign, the slant product of $[\Omega]_{n-k}$ with $\kappa$. We will use absolute value signs to show that we are neglecting sign. Thus, we may also denote $c_{k}(M) \kappa$ by

$$
|[\Omega] / \kappa| \text {. }
$$

In this paper we are concerned with the top class $c_{n}(M)$. We may express $[\Omega]_{0}$ as

$$
\alpha_{n} \otimes 1 \in H^{n}\left(B \text { Diff }_{\omega} M\right) \otimes H^{0}(M) \cong H^{n}\left(\bar{B} \text { Diff }_{\omega} M\right) \otimes \mathbf{R} \text {. }
$$

So we may identify $H^{n}\left(\bar{B} \mathscr{D}_{i f f} M\right)$ with $H^{n}\left(\bar{B} \mathscr{D}_{i f f_{\omega}} M\right) \otimes H^{0}(M)$ and thus identify $c_{n}(M)$ with $[\Omega]_{0}$. The class $[\Omega]_{0}$ is the same as $[\Omega]$ restricted to $\bar{B} \mathscr{D i f f}_{\omega} M \times \mathrm{pt}$. Therefore,

$$
c_{n}(M) \neq\left. 0 \Leftrightarrow[\Omega]\right|_{\bar{B} \text { aiff }_{\omega} M \times \mathrm{pt}} \neq 0 .
$$


Fundamental diagram. The diagram we use follows from [T]. Recall that $B \Gamma_{s l}^{n}$ is the classifying space for codimension- $n$ volume preserving Haefliger structures. There is a special cohomology class $\tilde{\mu} \in H^{n}\left(B \Gamma_{s l}^{n}\right)$ called the univeral transverse volume class. If $f: X \rightarrow B \Gamma_{s l}^{n}$ classifies a codimension- $n$ volume preserving Haefliger structure $H$, then $f^{*} \tilde{\mu} \in H^{n}(X)$ is called the transverse volume class of $H$. If $H$ is actually the Haefliger structure coming from a codimension- $n$ volume preserving foliation with transverse volume form $\lambda$, then $[\lambda]=f^{*} \tilde{\mu}$. The class $\tilde{\mu}$ can be constructed directly from the topological groupoid of germs $\Gamma_{s l}^{n}$, or it can be defined by a functorial principle.

Associated to $B \Gamma_{s l}^{n}$ we have the normal bundle of its universal Haefliger structure. This bundle can be classified by a map $d: B \Gamma_{s l}^{n} \rightarrow B \operatorname{Sl}(n, \mathbf{R})$. The map $d$ has a homotopy theoretic fibre $\bar{B} \Gamma_{s l}^{n}$. We choose models and maps so that we get an actual Hurewicz fibration

$$
\bar{B} \Gamma_{s l}^{n} \stackrel{i}{\rightarrow} B \Gamma_{s l}^{n} \stackrel{d}{\rightarrow} B \operatorname{Sl}(n, \mathbf{R})
$$

The space $\bar{B} \Gamma_{s l}^{n}$ is the classifying space for codimension- $n$ volume preserving Haefliger structures whose normal bundle is framed. This space is $(n-1)$-connected with $\pi_{n}\left(\bar{B} \Gamma_{s l}^{n}\right)=\mathbf{R}$. The class $i^{*} \tilde{\mu} \equiv \mu \in H^{n}\left(\bar{B} \Gamma_{s l}^{n}\right)$ corresponds to the identity homomorphism if we identify $H^{n}\left(\bar{B} \Gamma_{s l}^{n}\right)$ with $\operatorname{Hom}_{\mathbf{Z}}(\mathbf{R}, \mathbf{R})$ in the canonical manner.

The foliation $\mathscr{F}$ on $\bar{B} \mathscr{D i f f}_{\omega} M \times M$ can be classified by the map $\Phi$ into $B \Gamma_{s l}^{n}$. Consider the following diagram:

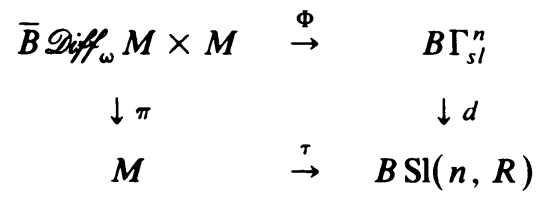

Here $\pi$ is projection, and $\tau$ classifies $T M$ (the choice of $\omega$ gives an Sl-structure). The normal bundle $\nu(\mathscr{F})$ of $\mathscr{F}$ is just $\bar{B} \mathscr{D i f f}_{\omega} M \times T M$ since $\mathscr{F}$ is transverse to the $M$-factors. We classify $\nu(\mathscr{F})$ by $d \circ \Phi$. Note that $\nu(\mathscr{F})$ is also classified by $\tau \circ \pi$. Everything is chosen so that (7) commutes and $d$ is a Hurewicz fibration.

We are interested in $\mathscr{L}(M)$, the space of lifts of $\tau$. Let us define $\Pi: \bar{B}$ Diff $_{\omega} M \rightarrow$ $\mathscr{L}(M)$ by setting $\Pi(b) \equiv \Phi(b, \cdot)$ for each $b \in \bar{B} \mathscr{D}_{i f f} M$. Since $M$ is compact and $n$-dimensional while the fibre of $d$ is $\bar{B} \Gamma_{s l}^{n}$, which is $(n-1)$-connected, the space $\mathscr{L}(M)$ is not connected. Because $\bar{B} \mathscr{D}_{i f f_{\omega}} M$ is connected, its image under $\Pi$ is in one component, $\mathscr{L}_{0}(M)$, of $\mathscr{L}(M)$. McDuff [M-2], in the spirit of Thurston [T], has shown that $\Pi^{*}$ is a cohomology isomorphism. This enables us to view $c_{n}(M)$ as living in $H^{n}\left(\mathscr{L}_{0}(M)\right)$. Let us exploit this philosophy.

Define $\varepsilon: \mathscr{L}_{0}(M) \rightarrow \bar{B} \Gamma_{s l}^{n}$ as evaluation at the fixed point $m_{0}$ in $M$. Consider $\mathscr{F} \mid$, $\mathscr{F}$ restricted to $\bar{B} \mathscr{D}_{i f} \mathcal{F}_{\omega} M \times m_{0}$. The bundle $\nu(\mathscr{F} \mid)$ is isomorphic to $\bar{B} \mathscr{D}_{\text {iff }} \boldsymbol{M} \times R^{n}$. This tell us that $\mathscr{F} \mid$ is a codimension-n Haefliger structure with trivial normal bundle. Due to this $\Phi \mid$, the map classifying $\mathscr{F} \mid$, is homotopic to a map with image in $\bar{B} \Gamma_{s l^{\prime}}^{n}$. Without loss of generality we may assume $\Phi \mid$ actually maps into $\bar{B} \Gamma_{s /}^{n}$. The following diagram homotopy commutes. 


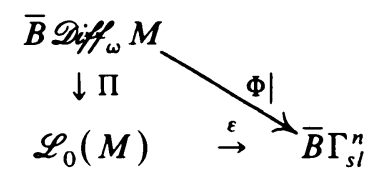

Recall that $c_{n}(M)$ is $\left.[\Omega]\right|_{\bar{B} \mathscr{C} f_{\omega}} M \times m_{0} \cdot$ Therefore, $\Phi^{*} \mu$ is $c_{n}(M)$. Since $\Pi^{*}$ is an isomorphism,

$$
c_{n}(M) \neq 0 \Leftrightarrow \varepsilon^{*} \mu \neq 0 .
$$

So the problem is now one of understanding the behavior of $\mathscr{L}_{0}(M) \stackrel{\varepsilon}{\rightarrow} \bar{B} \Gamma_{s l}^{n}$. Inherent in $\mathscr{L}_{0}(M)$ is the twisting of $T M$. The simpler that $T M$ is, the easier it is to understand $\varepsilon$. Such a case is provided when $M$ is stably parallelizable. By this we mean that $T M \oplus \varepsilon^{1} \cong \varepsilon^{n+1}$. The most obvious example of such a manifold is $S^{n}$. In fact spheres "classify" stably parallelizable manifolds.

(10) Proposition. The manifold $M$ is stably parallelizable if and only if $T M \cong$ $\gamma^{*}\left(T S^{n}\right)$ for some map $\gamma$ from $M \rightarrow S^{n}$.

Before proceeding further we need a technical lemma which follows easily from obstruction theory.

(11) LEMMA. If $f_{0}$ and $f_{1}$ are two lifts in $\mathscr{L}(M)$, they are in the same component of $\mathscr{L}(M)$ if and only if $f_{0}^{*} \tilde{\mu}=f_{1}^{*} \tilde{\mu}$.

Since $\Pi(b)$ is $\Phi(b, \cdot)$ and $\Phi(b, \cdot)^{*} \tilde{\mu}$ is $\left.[\Omega]\right|_{b \times M}=[\omega]$, we have

$$
f \in \mathscr{L}_{0}(M) \Leftrightarrow f^{*} \tilde{\mu}=[\omega] .
$$

(13) THEOREM. If $M$ is an odd-dimensional stably parallelizable manifold, then $c_{n}(M) \neq 0$.

REMARK. It is essential that $n$ be odd, for McDuff [M-1] has shown that $c_{2 n}\left(S^{2 n}\right)=0$ and $c_{2 n+1}\left(S^{2 n+1}\right) \neq 0$.

Proof. Case 1 . Suppose that $M$ is stably parallelizable but not parallelizable. Then by (10) $T M \cong \gamma^{*}\left(T S^{n}\right)$, and $\gamma$ has nonzero degree. Certainly we can give $M$ a volume form $\omega_{M}$ such that $\left[\omega_{M}\right]=\gamma^{*}\left[\omega_{S}\right]$, where $\omega_{S}$ is the standard volume form on $S^{n}$. Consider the following diagram.

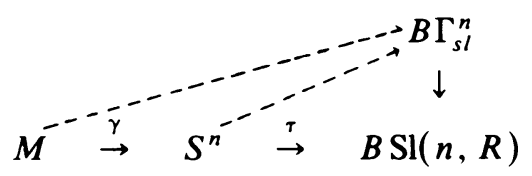

The map $\tau$ classifies $T S^{n}$ and therefore $\tau \circ \gamma$ classifies $T M$. Say $f \in \mathscr{L}_{0}\left(S^{n}\right)$; then $f^{*} \tilde{\mu}=\left[\omega_{S}\right]$. It follows that $f \circ \gamma \in \mathscr{L}_{0}(M)$. Hence, we have a map $\eta: \mathscr{L}_{0}\left(S^{n}\right) \rightarrow$ $\mathscr{L}_{0}(M)$ given by $\eta(f)=f \circ \gamma$. Consider the next diagram, where $\varepsilon_{m}$ is evaluation at $m_{0} \in M$ and $\varepsilon_{s}$ is evaluation at $\gamma\left(m_{0}\right) \varepsilon S^{n}$.

$$
\begin{array}{ccc}
\mathscr{L}_{0}\left(S^{n}\right) & \stackrel{\eta}{\rightarrow} & \mathscr{L}_{0}(M) \\
\downarrow \varepsilon_{s} & \swarrow \varepsilon_{m} & \\
\bar{B} \Gamma_{s l}^{n} &
\end{array}
$$


Since $\varepsilon_{m} \circ \eta(f)=\varepsilon_{m}(f \circ \gamma)=(f \circ \gamma)\left(m_{0}\right)=\varepsilon_{s} \circ f$, the above diagram commutes. Since $c_{n}\left(S^{n}\right) \neq 0$ for $n$ odd, (9) tells us that $\varepsilon_{s}^{*} \mu \neq 0$; therefore $\varepsilon_{n}^{*} \mu \neq 0$ and $c_{n}(M) \neq 0$.

Case 2. $M$ is parallelizable. Let $\tau$ now stand for a map classifying $T M$ into $B \mathrm{Sl}(n, R)$. Without loss of generality $\tau$ may be taken as a constant map. In this case (7) becomes

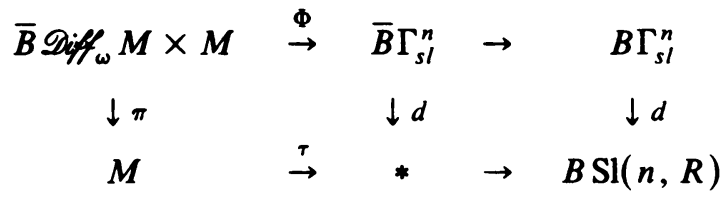

Since $M$ is parallelizable, $\mathscr{L}(M)=\operatorname{Maps}\left(M, \bar{B} \Gamma_{s l}^{n}\right)$ and we will designate the component corresponding to $\mathscr{L}_{0}(M)$ as $\operatorname{Maps}_{1}\left(M, \bar{B} \Gamma_{s l}^{n}\right)$. Therefore, $s \in$ $\operatorname{Maps}_{1}\left(M, \bar{B} \Gamma_{s l}^{n}\right)$ if and only if $s^{*} \mu=[\omega]$. The evaluation map $\varepsilon: \mathscr{L}_{0}(M) \rightarrow \bar{B} \Gamma_{s l}^{n}$ becomes $\varepsilon$ : $\operatorname{Maps}_{1}\left(M, \bar{B} \Gamma_{s l}^{n}\right) \rightarrow \bar{B} \Gamma_{s l}^{n}$. We wish to showthat $\varepsilon^{*} \mu \neq 0$. Choose $\omega$ so that $\langle[\omega],[M]\rangle=1$.

Following McDuff's proof $[\mathbf{M}-1]$ that $\pi_{n}\left(\bar{B} \Gamma_{s l}^{n}\right) \cong \mathbf{R}$, we choose a map $f: S^{n} \rightarrow \bar{B} \Gamma_{s l}^{n}$ such that $[f]=1 \in \pi_{n}\left(\bar{B} \Gamma_{s l}^{n}\right)$, i.e. $\left\langle f^{*} \mu,\left[S^{n}\right]\right\rangle=1$. Let us give $S^{n}$ a volume form $\omega_{S}$ so that $f^{*} \mu=\left[\omega_{S}\right]$. We will say that $g: M \rightarrow S^{n}$ is of degree one if $g^{*}\left[\omega_{S}\right]=[\omega]$. Let $\operatorname{Maps}_{1}\left(M, S^{n}\right)$ be all the maps of degree one. Now define a map $\hat{f}: \operatorname{Maps}_{1}\left(M, S^{n}\right)$ $\rightarrow \operatorname{Maps}_{1}\left(M, \bar{B} \Gamma_{s l}^{n}\right)$ by setting $\hat{f}(g)=f \circ g$. Let $\varepsilon$ and $\varepsilon^{\prime}$ be evaluation at $m_{0}$. The following diagram commutes:

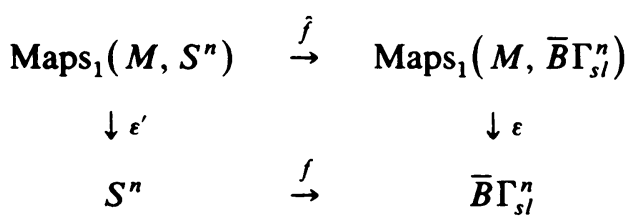

Since $f^{*} \mu=\left[\omega_{S}\right]$, if we can show that $\varepsilon^{\prime *}\left[\omega_{S}\right] \neq 0$, we will have shown that $\varepsilon^{*} \mu \neq 0$.

$\operatorname{Maps}_{1}\left(S^{n}, S^{n}\right)$ is the space of maps from $S^{n}$ to $S^{n}$ such that $f^{*}\left[\omega_{S}\right]=\left[\omega_{S}\right]$ (as before). Let $\xi$ be a fixed element of $\operatorname{Maps}_{1}\left(M, S^{n}\right)$ such that $\xi\left(m_{0}\right)=s$, the south pole of $S^{n}$. Define $\hat{\xi}: \operatorname{Maps}_{1}\left(S^{n}, S^{n}\right) \rightarrow \operatorname{Maps}_{1}\left(M, S^{n}\right)$ by $\hat{\xi}(h)=h \circ \xi$. The following diagram, with $\varepsilon^{\prime \prime}$ being evaluation at $s$, commutes.

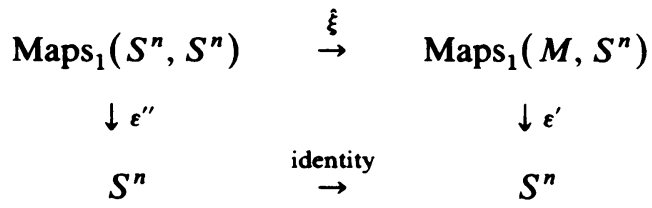

If we can show that $\varepsilon^{\prime *}\left[\omega_{S}\right] \neq 0$, then we will have shown that $\varepsilon^{\prime *}\left[\omega_{S}\right] \neq 0$ and we will be done. The left-hand side of (17) is from the fibration

$$
\Omega^{n} S_{1}^{n} \rightarrow \operatorname{Maps}_{1}\left(S^{n}, S^{n}\right) \rightarrow S^{n},
$$

where $\Omega^{n} S_{1}^{n}$ is the obvious component. Using the spectral sequence of (18) and the fact that $n$ is odd, we have $\varepsilon^{\prime \prime *}\left[\omega_{S}\right] \neq 0$. Q.E.D. 
We will now prove

(19) THEOREM. If $M$ is parallelizable then $c_{n}(M) \neq 0$.

REMARK. Here we have removed the condition of $n$ being odd but yet we still use $S^{n}$ in our argument.

Proof. Since $M$ is parallelizable it may be immersed in $\mathbf{R}^{n+1}[\mathbf{H}]$. We will consider $M$ as being immersed in $\mathbf{R}^{n+1}$ with a fixed immersion. We will also freely identify $M$ with its image in $\mathbf{R}^{n+1}$. This causes no trouble, as will become apparent. Our goal is to define a map $\Psi: M \rightarrow \operatorname{Maps}_{1}\left(M, S^{n}\right)$ such that if $\varepsilon^{\prime}$ is evaluation at $m_{0}$ then $\varepsilon^{\prime} \circ \Psi: M \rightarrow S^{n}$ has nonzero degree. If this is true then $\varepsilon^{\prime *}\left[\omega_{S}\right]$ is nonzero, where $\omega_{S}$ is the volume form on $S^{n}$. Let $\bar{\Psi}$ be the composite $\varepsilon^{\prime} \circ \Psi$.

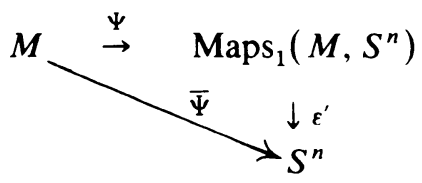

Choose positive $\varepsilon$ less than the injectivity radius of $M$ and small enough so that every (open) ball $B(p, \varepsilon)$ with center $p \in M$ and radius $\varepsilon$ is embedded by the immersion. Consider $\operatorname{Exp}^{-1}: B(p, \varepsilon) \rightarrow T M_{p}$. In fact, the image is in $B\left(O_{p}, \varepsilon\right) \subset T M_{p}$. Let $\gamma$ be the Gauss map from $M$ to $S^{n}$. By parallel translation in $\mathbf{R}^{n+1}$ we get a congruence from $T M_{p} \rightarrow T S_{\gamma(p)}^{n}$. Remembering that $\pi$ is the injectivity radius of the sphere we see that Exp maps $B\left(\vec{O}_{\gamma(p)}, \pi\right) \subset T S_{\gamma(p)}^{n}$ diffeomorphically onto $S^{n}-$ $A(\gamma(p))$, where $A$ is the antipodal map on $S^{n}$. Let $\zeta: T S^{n} \rightarrow T S^{n}$ by $\zeta(\vec{v})=\pi / \varepsilon \cdot \vec{v}$. We are now in a position to define $\Psi: M \rightarrow \operatorname{Maps}_{1}\left(M, S^{n}\right)$.

Decompose $M$ as $B(p, \varepsilon) \cup\{M-B(p, \varepsilon)\}$ :

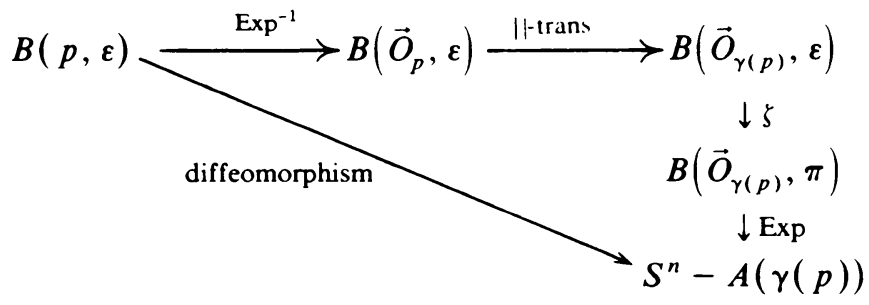

If $m \in B(p, \varepsilon)$ define $\Psi(p) m$ to be the image of $m$ under the above composition. At this stage we want the orientation on $B(p, \varepsilon)$, induced from the orientation of $M$, to go to the usual orientation on $S^{n}-A(\gamma(p))$. If it does not we give $M$ a different orientation and volume form. If $m \notin B(p, \varepsilon)$ set $\Psi(p) m \equiv A(\gamma(p))$.

As we vary $p$ we get a continuous map $\Psi: M \rightarrow \operatorname{Maps}_{1}\left(M, S^{n}\right)$. Each $\Psi(p)$ is of degree one, for $\Psi(p)$ is just a standard collapsing map of degree one.

(22) LEMMA. $\operatorname{deg} \bar{\Psi}=(-1)^{n+1} \operatorname{deg} \gamma+(-1)^{n}$, where deg stands for degree of the map.

Proof of Lemma. We just sketch the proof since the techniques are standard. If necessary, the first thing we do is adjust $\varepsilon$ in our definition of $\Psi$ to make sure that in a neighborhood $D$ containing $B\left(m_{0}, \varepsilon\right)$ we can slightly deform $M$ so that $D$ is flat. 
Thus, the Gauss map $\gamma$ is constant on $D$, hence $\gamma(D) \equiv \gamma\left(m_{0}\right)$. On $M-D, \bar{\Psi}$ sends $x$ to $\Psi(x) m_{0}=A(\gamma(x))$. If $x \in D$ then we must be careful. If $x \in D-B\left(M_{0}, \varepsilon\right)$, then $\bar{\Psi}(x)=\Psi(x) m_{0}=A(\gamma(x))=A\left(\gamma\left(m_{0}\right)\right)$ since $D$ is flat. The map $\bar{\Psi}$ restricted to $B\left(m_{0}, \varepsilon\right)$ has degree $(-1)^{n} . \bar{\Psi}$ is not smooth on $\overline{\partial B\left(m_{0}, \varepsilon\right)}$. Therefore, we replace it by a map $\bar{\Psi}^{\prime}$ in the same homotopy class and very close to $\bar{\Psi}$ and calculate the Brouwer degree. The $(-1)^{n+1} \operatorname{deg} \gamma$ part comes from $A \circ \gamma$, while $(-1)^{n}$ is due to the fact that $\bar{\Psi}$, near $m_{0}$, is a local diffeomorphism. By a judicious choice of a regular value it is easy to show that $\bar{\Psi}^{\prime}$, and hence $\bar{\Psi}$, has the proper degree. The proof of the lemma is complete.

Remember that we are trying to show that if $M$ is parallelizable then $c_{n}(M) \neq 0$. By (16) if we can show that $\varepsilon^{\prime *}\left[\omega_{s}\right] \neq 0$ we will be done. By the previous lemma $\bar{\Psi}$ has degree $(-1)^{n}+(-1)^{n+1} \operatorname{deg} \gamma$. If we can show that this is nonzero, then $\varepsilon^{\prime *}\left[\omega_{s}\right] \neq 0$. This is our plan. We will vary our immersion of $M$ so that we get deg $\gamma$ to our liking. To accomplish this we appeal to some results of Hopf [Ho, Mi].

(23) TheOREM (HOPF). Let L: $M^{n} \rightarrow \mathbf{R}^{n+1}$ be an immersion with corresponding Gauss map $\gamma(L): M^{n} \rightarrow S^{n}$.

(a) If $n$ is even, $\operatorname{deg} \gamma(L)=\frac{1}{2} \chi(M)$, where $\chi(M)$ is the Euler characteristic of $M$.

(b) If $n$ is odd and $\operatorname{deg} \gamma(L)=k$, then given any $m$ one can find an immersion $j=j(m)$ such that $\operatorname{deg} \gamma(j)=k+2 m$.

Thus $\bar{\Psi}$ can be taken to have nonzero degree. Q.E.D.

REMARK. It is worth pointing out that all 3-manifolds and products of spheres with one factor being odd are parallelizable and therefore have $c_{n}(M) \neq 0$.

\section{REFERENCES}

[H] M. Hirsch, Immersions of manifolds, Trans. Amer. Math. Soc. 93 (1959), 242-276.

. [Ho] H. Hopf, Über die Curvature integra geschlossener Hyperflächen, Math. Ann. 95 (1925/26), 340-367.

[L] C. C. Liang, On the volume-preserving foliations, Math. Ann. 223 (1976), 13-17.

[Ma] J. N. Mather, On the homology of Haefliger's classifying space, Carenna (1976).

[M-1] D. McDuff, Some canonical cohomology classes on groups of volume preserving diffeomorphisms, Trans. Amer. Math. Soc. 275 (1983), 345-356.

[M-2] __ Local homology of groups of volume preserving diffeomorphisms. III (to appear).

[Mi] J. Milnor, On the immersion of n-manifolds in $(n+1)$-space, Comment. Math. Helv. 30 (1956), 275-284.

[Mo] I. Moskowitz, Volume preserving foliations and diffeomorphism groups, Ph.D. dissertation, SUNY at Stony Brook, 1983.

[T] W. Thurston, Foliations and groups of diffeomorphisms, Bull. Amer. Math. Soc. 80 (1974), 304-307.

Department of Mathematics, Texas A \& M University, College Station, TeXas 77843 